\title{
Generalized Napoly integral to compute wake potentials in axisymmetric structure
}

\author{
Yoshihiro Shobuda \\ JAEA, Japan Atomic Energy Agency, 2-4 Shirakata Shirane, Tokaimura, Nakagun, Ibaraki 319-1195, Japan \\ Yong Ho Chin and Koji Takata \\ KEK, High Energy Accelerator Research Organization, 1-1 Oho, Tsukuba, Ibaraki 305-0801, Japan
}

(Received 30 August 2007; published 28 January 2008)

\begin{abstract}
The Napoly integral is the very useful method for calculations of wake potentials in structures where parts of the boundary extend below the beam pipe radius or the radii of the two beam pipes at both ends are unequal. It reduces CPU time a lot by deforming the integration path so that the integration contour is confined to the finite length over the gap of the structures. However, the original Napoly method cannot be applied to the transverse wake potentials in a structure where the two beam tubes on both sides have unequal radii . In this case, the integration path needed to be a straight line and the integration is stretched out to an infinite, in principle. We generalize the Napoly integrals so that integrals are always confined in a finite length even when the two beam tubes have unequal radii, for both longitudinal and transverse wake potential calculations. The extended method has been successfully implemented to the ABCI code.
\end{abstract}

DOI: 10.1103/PhysRevSTAB.11.011003

PACS numbers: 29.27.Bd, 02.60.Cb

\section{INTRODUCTION}

Calculation of wake potentials in accelerators is done mostly using simulation codes such as ABCI [1] which solve the Maxwell equations for given structures. The conventional integration method for the calculation of wake potentials at the radius of the beam pipe breaks down when parts of the structures extend below the beam pipe radius such as collimators. In these cases, the integration path needs to be moved closer to the beam axis so that the contour does not intersect the structure. Then, a very long outgoing beam pipe may become necessary to allow the wakefields to catch up with the beam far beyond the structure. This may require excessive computer memory and CPU time.

The Napoly integration method was a solution for this classical problem of wake potential calculations in these structures [2,3]. In their original method, the integration along the longitudinal direction on a straight line can be replaced by an integral along a contour beginning and ending on beam pipes by applying the Napoly-Zotter contour [3]. This is because the contribution of the radiated fields from $z= \pm \infty$ and the source fields become zero for this specific structure. For the monopole wake potential case, this method permits also a structure with unequal beam pipe radii at both ends by adding the contribution from the source fields (this was already done by themselves, see Appendix A), because the contribution of the radiated fields from $z= \pm \infty$ is still zero in this monopole mode. This method is very useful for the numerical calculations, where the electromagnetic (EM) fields satisfying all the boundary conditions are known.

In the axisymmetric structure, we develop the general theory based on the mathematical rigidity. Actually, this generalization of the method of Napoly et al. should be indispensable, because their discussion is focused on the case that the radii of the two beam pipes are equal for higher order mode wake potential. In other words, for the calculation of wake potentials of higher than the dipole mode, their method fails when the radii of the two beam pipes are unequal. It is necessary to consider the contribution of the radiated fields from $z= \pm \infty$ and the source fields more carefully for this complicated case, differently from the monopole case.

Another approach has been made by Henke and Bruns [4] to calculate wake potentials for three-dimensional structures. They assume that $H_{z}=0$ in their formalism and pick up only the TM and TEM fields from the Maxwell equations. They found the irrotational vector under this assumption and derived the formula for wake potentials in the Cartesian coordinate using the property of this vector. It is, however, not clear whether this extraction of the TM and TEM fields only from the Maxwell equations is enough to create an accurate formalism.

On the contrary, our method makes no such assumption regarding the Maxwell equations to construct the formalism. Thus, the final formula is an exact solution. In addition, because of our dealing with axisymmetrical structures, the boundary conditions on both beam pipe ends and the potential different effects in the case of unequal beam pipe radii on both ends are explicitly expressed in the final formula and they can be easily evaluated.

In this paper, we generalize Napoly's method for any axisymmetric structure and for any mode, including a structure when the radii of the two beam pipes are unequal. The basic concept of our calculation is introduced in Sec. II. In Sec. III A, we review Napoly's derivation of the expression of wake potential for modes higher than the dipole mode for a structure with equal beam pipe radii at 
both ends. Then, we derive formulas for wake potentials for any type of structure by generalizing their method in Sec. III B. The extended method has been implemented to ABCI code. We demonstrate the usefulness of the present method in Sec. IV by comparing numerical results with ones obtained by the conventional integration method. The paper is summarized in Sec. V.

\section{THE BASIC CONCEPT}

The goal of this paper is to find an appropriate integration contour, which starts and ends on the two beam pipes, and combinations of EM fields to be integrated along this integration contour. For this purpose, we need to make some preparations for later manipulations. If there is a vector whose loop integration is zero, and we need to integrate it along a part of this loop, we are able to deform this integration path by using this property. Our first task is to find such a vector. It is convenient to describe this point in terms of the differential form. We assume the one-form: $\omega=A_{r} d r+A_{z} d z$ on the contractible space: $R^{2}$. If the oneform is closed, namely $d \omega=0$, there exists $f$ that satisfies $\omega=d f$. This is known as Poincaré's lemma. We use this useful feature for deforming of the integration path for wake potential calculations. Following Napoly et al. [3], we introduce such one-form for an axisymmetric structure.

We use the cylindrical coordinate $(r, \theta, z)$, and assume that the source particle is located at $\vec{r}_{0}=\left(r_{0}, \theta_{0}=0\right)$. Because of the axisymmetry of the structure, the electromagnetic fields can be Fourier expanded in the azimuthal direction as follows:

$$
\begin{aligned}
& \left(E_{r}, B_{\theta}, E_{z}\right)(r, \theta, z, t)=\sum_{m=0}^{\infty}\left(e_{r}, b_{\theta}, e_{z}\right)^{(m)}(r, z, t) \cos m \theta \\
& \left(B_{r}, E_{\theta}, B_{z}\right)(r, \theta, z, t)=\sum_{m=1}^{\infty}\left(b_{r}, e_{\theta}, b_{z}\right)^{(m)}(r, z, t) \sin m \theta
\end{aligned}
$$

The electromagnetic fields can be decomposed as $E=$ $E^{(s)}+E^{(r)}$ and $B=B^{(s)}+B^{(r)}$, where $\left(E^{(s)}, B^{(s)}\right)$ are the source fields and $\left(E^{(r)}, B^{(r)}\right)$ are the radiated fields (which are the solutions of homogeneous Maxwell equations).

Let us introduce the following definition for a generic field:

$$
\bar{\phi}(z, s) \equiv \phi[z, t(z, s)],
$$

in such a way that

$$
\partial_{z} \bar{\phi}(z, s)=\left(\partial_{z}+\partial_{c t}\right) \phi[z, t(z, s)]
$$

where $s$ is the distance between a test particle and a given origin of the exciting bunch $z_{0}=c t$, and $t(z, s)=(z+$ $s) / c$.
The Maxwell equations for the radiated fields are described as

$$
\begin{gathered}
\partial_{r} \bar{E}_{z}^{(r)}-\partial_{z}\left(\bar{E}_{r}^{(r)}+c \bar{B}_{\theta}^{(r)}\right)=-\frac{1}{r} \partial_{\theta}\left(c \bar{B}_{z}^{(r)}\right), \\
\partial_{r}\left(c \bar{B}_{z}^{(r)}\right)+\partial_{z}\left(\bar{E}_{\theta}^{(r)}-c \bar{B}_{r}^{(r)}\right)=\frac{1}{r} \partial_{\theta} \bar{E}_{z}^{(r)} .
\end{gathered}
$$

By substituting Eqs. (1) and (2) into Eqs. (5) and (6), they can be rewritten as

$$
\begin{aligned}
{\left[\partial_{r} \bar{e}_{z}^{(r)}-\partial_{z}\left(\bar{e}_{r}^{(r)}+c \bar{b}_{\theta}^{(r)}\right)\right]^{(m)} } & =-\frac{m}{r}\left[c \bar{b}_{z}^{(r)}\right]^{(m)}, \\
{\left[\partial_{r}\left(c \bar{b}_{z}^{(r)}\right)+\partial_{z}\left(\bar{e}_{\theta}^{(r)}-c \bar{b}_{r}^{(r)}\right)\right]^{(m)} } & =-\frac{m}{r}\left[\bar{e}_{z}^{(r)}\right]^{(m)} .
\end{aligned}
$$

We define two one-forms: $\omega_{1}=S_{r}^{(m)} d r+S_{z}^{(m)} d z$ and $\omega_{2}=D_{r}^{(m)} d r+D_{z}^{(m)} d z$, where

$$
\begin{aligned}
S^{(m)}= & \left(r^{m}\left[\bar{e}_{r}^{(r)}+c \bar{b}_{\theta}^{(r)}-\bar{e}_{\theta}^{(r)}+c \bar{b}_{r}^{(r)}\right]^{(m)},\right. \\
& \left.r^{m}\left[\bar{e}_{z}^{(r)}+c \bar{b}_{z}^{(r)}\right]^{(m)}\right), \\
D^{(m)}= & \left(r^{-m}\left[\bar{e}_{r}^{(r)}+c \bar{b}_{\theta}^{(r)}+\bar{e}_{\theta}^{(r)}-c \bar{b}_{r}^{(r)}\right]^{(m)},\right. \\
& \left.r^{-m}\left[\bar{e}_{z}^{(r)}-c \bar{b}_{z}^{(r)}\right]^{(m)}\right),
\end{aligned}
$$

in the $(r, z)$-plane [3]. From Eqs. (7) and (8), it can be found that the components of each vector $S^{(m)}$ and $D^{(m)}$ satisfy

$\partial_{r}\left(r^{m}\left[\bar{e}_{z}^{(r)}+c \bar{b}_{z}^{(r)}\right]^{(m)}\right)=r^{m} \partial_{z}\left[\bar{e}_{r}^{(r)}+c \bar{b}_{\theta}^{(r)}-e_{\theta}^{(r)}+c \bar{b}_{r}^{(r)}\right]^{(m)}$,

$$
\begin{aligned}
& \partial_{r}\left(r^{-m}\left[\bar{e}_{z}^{(r)}-c \bar{b}_{z}^{(r)}\right]^{(m)}\right) \\
& =r^{-m} \partial_{z}\left[\bar{e}_{r}^{(r)}+c \bar{b}_{\theta}^{(r)}+e_{\theta}^{(r)}-c \bar{b}_{r}^{(r)}\right]^{(m)} .
\end{aligned}
$$

These equations indicate that both one-forms, $\omega_{1}$ and $\omega_{2}$, are closed. Since the $(r, z)$-plane is contractible, there is a scalar quantity whose differential provides $S^{(m)}$ and $D^{(m)}$. Therefore, integrals of $S^{(m)}$ and $D^{(m)}$ along any closed contour (loop) in $(r, z)$-plane vanish. The next step is to express wake potentials using $S^{(m)}$ and $D^{(m)}$ and to replace their integrations along a straight line to those on an arbitrary contour in such a way that the straight line and the contour compose a loop.

\section{LONGITUDINAL WAKE POTENTIALS OF DIPOLE OR HIGHER ORDER MODE $(m>0)$}

Napoly et al. derived the formula for these modes, but its application is limited to structures with beam pipes of equal radius on both sides. Their point of justification for this limitation is that "the method (in the case of unequal beam tube radii) is not much better than the direct wakefield integration along a straight line $\mathcal{L}_{r}$." However, there is a contribution to wake potentials from the potential 
difference of EM fields in the two beam pipes and one has to subtract this contribution from results of the direct wakefield integration in order to get pure effects of radiated fields. Since no hint is given in their paper on how to calculate the contribution of the potential difference of EM fields, neither their formula nor the direct integration method can provide correct wake potentials in the case of unequal beam tube radii, anyway. In this section, we expand their formula for any mode and for any structure, including structures with beam pipes of unequal radii on both sides. In Sec. III A, we follow their procedure and review their formula to understand how the integration path can be deformed. In Sec. III B, we generalize Napoly's method to the case that the radii of both ends of the beam tube are unequal.

\section{A. Beam pipes of equal radii on both ends}

Let us consider an axisymmetric cavity as shown in Fig. 1. Here we assume that the radius of the beam tube on the downstream side $a_{\text {out }}$ is equal to that on the upstream side $a_{\text {in }}\left(=a_{\text {out }}=a\right)$. A closed loop $G$ (see the right part of Fig. 1) is defined in such a way that it consists of a straight line $\mathcal{L}_{r}$ and Napoly-Zotter contour $\overline{\mathcal{C}}$ and the two radial lines connecting between them at $z= \pm \infty$. The longitudinal coordinate $z_{1}$ and $z_{2}$, and the radial coordinate $a_{0}$ describe the Napoly-Zotter contour $\overline{\mathcal{C}}$.

The integration of the vectors $S^{(m)}$ and $D^{(m)}$ over $G$ vanishes. Since the radial offsets of the contour $\overline{\mathcal{C}}$ at $z=$ $\infty$ and $z=-\infty$ are equal, the $r$-integrations of radiated field at $z=-\infty$ and $z=\infty$ cancel each other out. Then, we obtain the following identities:

$$
\begin{aligned}
& \int_{\mathcal{L}_{r}} S^{(m)}(r, \tilde{z}, s) d \tilde{l}=\int_{\overline{\mathcal{C}}} S^{(m)}\left(r^{\prime}, z^{\prime}, s\right) d l^{\prime}, \\
& \int_{\mathcal{L}_{r}} D^{(m)}(r, z, s) d l=\int_{\overline{\mathcal{C}}} D^{(m)}\left(r^{\prime}, z^{\prime}, s\right) d l^{\prime} .
\end{aligned}
$$

By taking the contour $\mathcal{L}_{r}$ to be the beam chamber axis $\mathcal{L}_{0}$ or the contour $\overline{\mathcal{C}}$ to be the beam chamber axis $\mathcal{L}_{0}$, we obtain

$$
\begin{gathered}
\int_{\overline{\mathcal{C}}} S^{(m)}\left(r^{\prime}, z, s\right) d l=0, \\
\int_{-\infty}^{\infty}\left[\bar{e}_{z}^{(r)}(r, z, s)\right]^{(m)} d z=-\int_{-\infty}^{\infty}\left[c \bar{b}_{z}^{(r)}(r, z, s)\right]^{(m)} d z,
\end{gathered}
$$

respectively. by

The longitudinal wake potential for mode $m$ is defined

$$
W_{z}^{(m)}(r, \theta, s) \equiv-\frac{1}{Q} \int_{-\infty}^{\infty} d z E_{z}[r, \theta, z, t(z, s)],
$$

where $Q$ is total charge, and the longitudinal charge distribution $\lambda(s)$ is normalized as

$$
\int_{-\infty}^{\infty} \lambda(s) d s=1
$$

The transverse wake potential is defined by

$$
W_{\perp}^{(m)}(r, \theta, s) \equiv-\frac{1}{Q} \int_{-\infty}^{\infty} d z\left(E_{\perp}+v \times B\right)[r, \theta, z, t(z, s)] .
$$

Transverse wake potentials can be obtained from longitudinal ones of the same mode using the Panofsky-Wenzel theorem as [5]

$$
\partial_{s} W_{\perp}^{(m)}(r, \theta, s)=\nabla_{\perp} W_{z}^{(m)}(r, \theta, s) .
$$

Since the source term of the longitudinal electric field is zero, Eq. (17) can be expressed by the integration of the radiated field $\left[\bar{e}_{z}^{(r)}\right]^{(m)}$ only:

$$
\begin{aligned}
W_{z}^{(m)}(r, \theta, s) & =-\frac{\cos m \theta}{Q} \int_{-\infty}^{\infty} d z^{\prime}\left[\bar{e}_{z}^{(r)}\right]^{(m)} \\
& =-\frac{\cos m \theta}{2 Q} \int_{-\infty}^{\infty} d z^{\prime}\left(\left[\bar{e}_{z}^{(r)}\right]^{(m)}+\left[\bar{e}_{z}^{(r)}\right]^{(m)}\right) .
\end{aligned}
$$

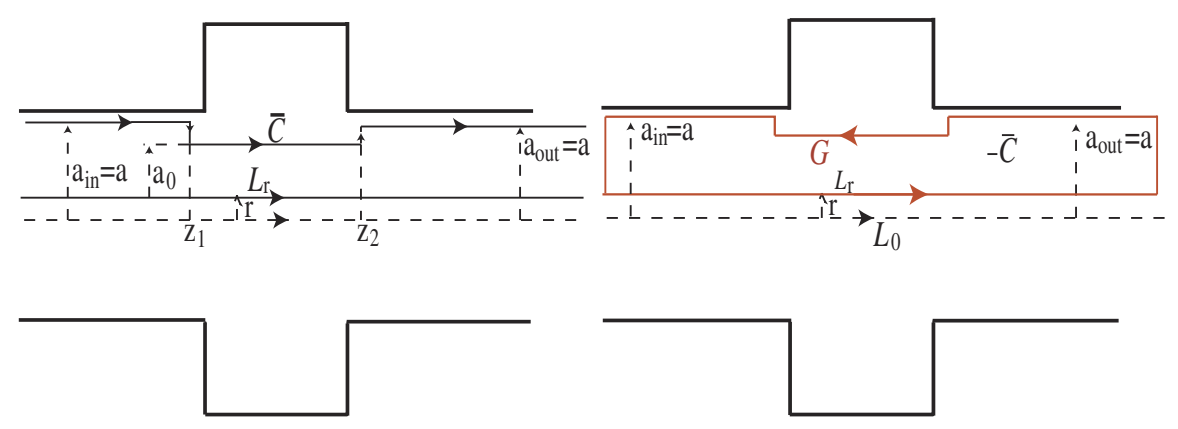

FIG. 1. (Color) The cavity and the integration path. Here we assume that the radius of the beam tube on the downstream side $a_{\text {out }}(=a)$ is equal to that on the upstream side $a_{\text {in }}(=a)$. The longitudinal coordinate $z_{1}$ and $z_{2}$, and the radial coordinate $a_{0}$ specify the contour $\overline{\mathcal{C}}$ (Napoly-Zotter contour [3]). The contour $\mathcal{L}_{r}$ is the straight line of radius $r$. A closed loop $G$ is defined in such a way that it consists of a straight line $\mathcal{L}_{r}$ and Napoly-Zotter contour $\overline{\mathcal{C}}$ and the two radial lines connecting between them at $z= \pm \infty$. The sign of contour $-\overline{\mathcal{C}}$ means that the direction of the integration path is opposite to $\overline{\mathcal{C}}$. 
This expression can be further rewritten by substituting Eq. (16) into one of $\left[\bar{e}_{z}^{(r)}\right]^{(m)}$ as

$$
W_{z}^{(m)}(r, \theta, s)=-\frac{r^{m} \cos m \theta}{2 Q} \int_{-\infty}^{\infty} d z^{\prime} r^{-m}\left(\left[\bar{e}_{z}^{(r)}\right]^{(m)}-c\left[\bar{b}_{z}^{(r)}\right]^{(m)}\right)=-\frac{r^{m} \cos m \theta}{2 Q} \int_{\mathcal{L}_{r}} d z^{\prime} D^{(m)}\left(r, z^{\prime}, s\right) .
$$

Here we used Eq. (10) to rewrite the first line to the second line. The integration path $\mathcal{L}_{r}$ can be deformed to the NapolyZotter contour $\overline{\mathcal{C}}$ by using Eq. (14). Since Eq. (15) shows that the integration of $S^{(m)}$ over the contour $\overline{\mathcal{C}}$ is zero, we can add this term to Eq. (22). We then obtain

$$
\begin{aligned}
W_{z}^{(m)}(r, \theta, s)= & -\frac{\cos m \theta}{2 Q} r^{m}\left(\int_{\overline{\mathcal{C}}} D^{(m)}\left(r^{\prime}, z^{\prime}, s\right) d l^{\prime}+\frac{1}{a^{2 m}} \int_{\overline{\mathcal{C}}} S^{(m)}\left(r^{\prime}, z^{\prime}, s\right) d l^{\prime}\right) \\
= & -\frac{\cos m \theta}{2 Q} \frac{r^{m}}{a^{m}}\left\{\int_{\overline{\mathcal{C}}} d z^{\prime}\left[\bar{e}_{z}^{(r)}\left(\frac{a^{m}}{r^{\prime m}}+\frac{r^{\prime m}}{a^{m}}\right)-c \bar{b}_{z}^{(r)}\left(\frac{a^{m}}{r^{\prime m}}-\frac{r^{\prime m}}{a^{m}}\right)\right]^{(m)}\right. \\
& \left.+\int_{\overline{\mathcal{C}}} d r^{\prime}\left[\left(\bar{e}_{r}^{(r)}+c \bar{b}_{\theta}^{(r)}\right)\left(\frac{a^{m}}{r^{\prime m}}+\frac{r^{\prime m}}{a^{m}}\right)+\left(\bar{e}_{\theta}^{(r)}-c \bar{b}_{r}^{(r)}\right)\left(\frac{a^{m}}{r^{\prime m}}-\frac{r^{\prime m}}{a^{m}}\right)\right]^{(m)}\right\} .
\end{aligned}
$$

Let us be reminded that Eq. (13) is obtained under the condition that the contributions of the integration at $z= \pm \infty$ cancel each other out since the radii of the beam pipes on the two sides are equal.

For numerical evaluation, it is necessary to describe Eq. (23) by the real fields instead of the radiated fields. The source fields do not contribute to the results, because the longitudinal components are zero and the transverse components depend only on $r^{\prime}$ and $s$ (here we use that the contour $\overline{\mathcal{C}}$ is symmetric). The longitudinal wake potential is expressed by

$$
\begin{aligned}
W_{z}^{(m)}(r, \theta, s)= & -\frac{\cos m \theta}{2 Q} \frac{r^{m}}{a^{m}}\left\{\int_{\overline{\mathcal{C}}} d z^{\prime}\left[\bar{e}_{z}\left(\frac{a^{m}}{r^{\prime m}}+\frac{r^{\prime m}}{a^{m}}\right)-c \bar{b}_{z}\left(\frac{a^{m}}{r^{\prime m}}-\frac{r^{\prime m}}{a^{m}}\right)\right]^{(m)}\right. \\
& \left.+\int_{\overline{\mathcal{C}}} d r^{\prime}\left[\left(\bar{e}_{r}+c \bar{b}_{\theta}\right)\left(\frac{a^{m}}{r^{\prime m}}+\frac{r^{\prime m}}{a^{m}}\right)+\left(\bar{e}_{\theta}-c \bar{b}_{r}\right)\left(\frac{a^{m}}{r^{\prime m}}-\frac{r^{\prime m}}{a^{m}}\right)\right]^{(m)}\right\} .
\end{aligned}
$$

The integral is confined to the region $-z_{1}<z<z_{2}$, because the longitudinal electric field is zero on the surface of the chamber.

\section{B. Beam pipes of unequal radii on both ends}

When we derive Eq. (24), we use the facts that the contribution from the radiated field at both ends of the beam tube cancel each other out and that the integral of the source field over the contour $\overline{\mathcal{C}}$ does not contribute to the wake potential. When the radii of both sides of the beam tube are unequal, we need to consider these contributions more carefully. For simplicity, we assume in this section that the radius of the beam tube on the downstream side $a_{\text {out }}$ is smaller than that on the upstream side $a_{\text {in }}$ [this constraint will be removed in the final expression of the wake potential, i.e., Eq. (51)]. The cavity and the integration path are shown in Fig. 2.

In order to evaluate the contributions of the radiated fields from $z= \pm \infty$, we need to know their asymptotic expressions at $z= \pm \infty$. The potentials of the fields inside a perfectly conducting pipe with the radius $a$ (which can be

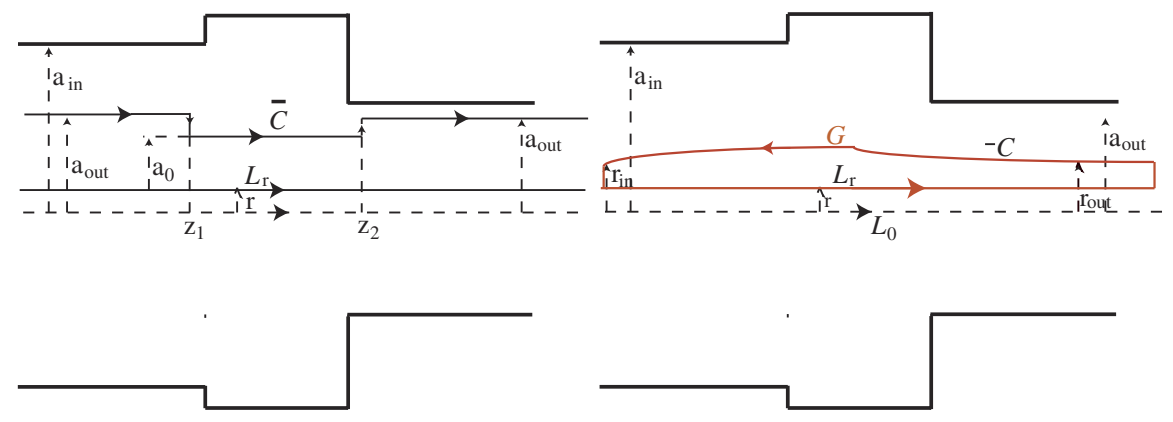

FIG. 2. (Color) The cavity and the integration path. Here we assume that the radius of the beam tube on the downstream side $a_{\text {out }}$ is smaller than that on the upstream side $a_{\text {in }}$. The contour $\mathcal{L}_{r}$ is the straight line of radius $r$. The contour $\mathcal{C}$ is arbitrary and this has radial offsets $r_{\text {in }}$ and $r_{\text {out }}$ at $z= \pm \infty$, respectively. A loop $G$ consists of a straight line $\mathcal{L}_{r}$ and arbitrary contour $\mathcal{C}$ and two radial lines connecting between them. The sign of contour $-\mathcal{C}$ means that the direction of the integration path is opposite to $\mathcal{C}$. We call the contour $\overline{\mathcal{C}}$ Napoly-Zotter contour. The longitudinal coordinate $z_{1}$ and $z_{2}$, and the radial coordinate $a_{0}$ specify the contour $\overline{\mathcal{C}}$. 
$a_{\text {in }}$ on the upstream side chamber and $a_{\text {out }}$ on the downstream side chamber) are given by (the radial and azimuthal component of the vector potentials are always zero in this paragraph)

$$
\begin{aligned}
\phi(r, \theta, z, t)= & \frac{Q}{2 \pi \epsilon_{0}} \lambda(c t-z)\left\{\log \left(\frac{a}{r_{>}}\right)\right. \\
& \left.+\sum_{m=1}^{\infty} \frac{\cos m \theta}{m}\left[\left(\frac{r_{<}}{r_{>}}\right)^{m}-\left(\frac{r r_{0}}{a^{2}}\right)^{m}\right]\right\}, \\
& A_{z}(r, \theta, z, t)=\phi(r, \theta, z, t) / c,
\end{aligned}
$$

where $r_{>}=\sup \left(r, r_{0}\right)$ and $r_{<}=\inf \left(r, r_{0}\right)$. Here we assume that the source fields are defined as

$$
\begin{gathered}
\phi^{(s)}(r, \theta, z, t)=\frac{Q}{2 \pi \epsilon_{0}} \lambda(c t-z)\left[\log \left(\frac{a_{\mathrm{out}}}{r_{>}}\right)\right. \\
\left.+\sum_{m=1}^{\infty} \frac{\cos m \theta}{m}\left(\frac{r_{<}}{r_{>}}\right)^{m}\right], \\
A_{z}^{(s)}(r, \theta, z, t)=\phi^{(s)}(r, \theta, z, t) / c .
\end{gathered}
$$

By subtracting the source fields [Eqs. (27) and (28)] from the potentials [Eqs. (25) and (26)], the radiated fields $(E, B)^{(r)}$ for $z \rightarrow-\infty$ are given by

$$
\begin{gathered}
\bar{\phi}_{\text {in }}^{(r)}=\frac{Q}{2 \pi \epsilon_{0}} \lambda(s)\left[\log \left(\frac{a_{\text {in }}}{a_{\text {out }}}\right)-\sum_{m=1}^{\infty} \frac{\cos m \theta}{m}\left(\frac{r r_{0}}{a_{\text {in }}^{2}}\right)^{m}\right], \\
\bar{A}_{z, \text { in }}^{(r)}=\bar{\phi}_{\text {in }}^{(r)} / c
\end{gathered}
$$

and those for $z \rightarrow \infty$ are derived from

$$
\begin{gathered}
\bar{\phi}_{\text {out }}^{(r)}=-\frac{Q}{2 \pi \epsilon_{0}} \lambda(s) \sum_{m=1}^{\infty} \frac{\cos m \theta}{m}\left(\frac{r r_{0}}{a_{\text {out }}^{2}}\right)^{m}, \\
\bar{A}_{z, \text { out }}^{(r)}=\bar{\phi}_{\text {out }}^{(r)} / c .
\end{gathered}
$$

The EM fields are calculated from these potentials as

$$
\begin{gathered}
\bar{E}_{r}(z, s)=-\partial_{r} \bar{\phi}(z, s), \quad \bar{E}_{\theta}(z, s)=-\frac{1}{r} \partial_{\theta} \bar{\phi}(z, s), \\
\bar{E}_{z}(z, s)=-\partial_{z} \bar{\phi}(z, s), \\
c \bar{B}_{r}(z, s)=\frac{1}{r} \partial_{\theta} \bar{\phi}, \quad c \bar{B}_{r}(z, s)=-\partial_{r} \bar{\phi}, \\
c \bar{B}_{z}(z, s)=0,
\end{gathered}
$$

where $\bar{\phi}$ is either $\bar{\phi}_{\text {in }}$ or $\bar{\phi}_{\text {out }}$. The asymptotic forms of the radiated fields are summarized as

$$
\begin{aligned}
& \lim _{z \rightarrow-\infty} \bar{e}_{r}^{(r)}(r, z, s)=-\lim _{z \rightarrow-\infty} \bar{e}_{\theta}^{(r)}(r, z, s)=\lim _{z \rightarrow-\infty} c \bar{b}_{r}^{(r)}(r, z, s) \\
&=\lim _{z \rightarrow-\infty} c \bar{b}_{\theta}^{(r)}(r, z, s) \\
&=\frac{Q}{2 \pi r \epsilon_{0}} \lambda(s) \sum_{m=1}^{\infty}\left(\frac{r r_{0}}{a_{\mathrm{in}}^{2}}\right)^{m} \\
& \lim _{z \rightarrow-\infty} \bar{e}_{z}^{(r)}(r, z, s)=\lim _{z \rightarrow-\infty} c \bar{b}_{z}^{(r)}(r, z, s)=0 \\
& \lim _{z \rightarrow \infty} \bar{e}_{r}^{(r)}(r, z, s)=-\lim _{z \rightarrow \infty} \bar{e}_{\theta}^{(r)}(r, z, s)=\lim _{z \rightarrow \infty} c \bar{b}_{r}^{(r)}(r, z, s) \\
&=\lim _{z \rightarrow \infty} c \bar{b}_{\theta}^{(r)}(r, z, s) \\
&=\frac{Q}{2 \pi r \epsilon_{0}} \lambda(s) \sum_{m=1}^{\infty}\left(\frac{r r_{0}}{a_{\mathrm{out}}^{2}}\right)^{m}, \\
& \lim _{z \rightarrow \infty} \bar{e}_{z}^{(r)}(r, z, s)=\lim _{z \rightarrow \infty} c \bar{b}_{z}^{(r)}(r, z, s)=0
\end{aligned}
$$

We choose a closed loop $G$ (see the right part of Fig. 2) in such a way that it consists of a straight line $\mathcal{L}_{r}$ and an arbitrary contour $\mathcal{C}$ and the two radial lines connecting between them at $z= \pm \infty$. The contour $\mathcal{C}$ has radial offsets $r_{\text {in }}$ and $r_{\text {out }}$ at $z= \pm \infty$, respectively. The loop integrations of the vectors $S^{(m)}$ and $D^{(m)}$ over $G$ vanish. The integrations at $z= \pm \infty$ can be done using Eqs. (35)-(38). Then, we obtain

$$
\begin{aligned}
& \int_{\mathcal{L}_{r}} S^{(m)}(r, \tilde{z}, s) d \tilde{l}= \int_{\mathcal{C}} S^{(m)}\left(r^{\prime}, z^{\prime}, s\right) d l^{\prime} \\
&+\frac{Q}{\pi \epsilon_{0}} \lambda(s) \frac{r_{0}^{m}}{m}\left(\frac{r_{\mathrm{in}}^{2 m}}{a_{\mathrm{in}}^{2 m}}-\frac{r_{\mathrm{out}}^{2 m}}{a_{\mathrm{out}}^{2 m}}\right) \\
&-\frac{Q}{\pi \epsilon_{0}} \lambda(s) \frac{r_{0}^{m} r^{2 m}}{m}\left(\frac{1}{a_{\mathrm{in}}^{2 m}}-\frac{1}{a_{\mathrm{out}}^{2 m}}\right), \\
& \int_{\mathcal{L}_{r}} D^{(m)}(r, z, s) d l=\int_{\mathcal{C}} D^{(m)}\left(r^{\prime}, z^{\prime}, s\right) d l^{\prime},
\end{aligned}
$$

instead of Eqs. (13) and (14). By taking the contour $\mathcal{L}_{r}$ to be the axis $\mathcal{L}_{0}$, or the contour $\mathcal{C}$ to be the axis $\mathcal{L}_{0}$, we obtain

$$
\begin{aligned}
\int_{\mathcal{C}} S^{(m)}\left(r^{\prime}, z, s\right) d l=-\frac{Q}{\pi \epsilon_{0}} \lambda(s) \frac{r_{0}^{m}}{m}\left(\frac{r_{\mathrm{in}}^{2 m}}{a_{\mathrm{in}}^{2 m}}-\frac{r_{\mathrm{out}}^{2 m}}{a_{\mathrm{out}}^{2 m}}\right), & \\
\int_{-\infty}^{\infty}\left[\bar{e}_{z}^{(r)}(r, z, s)\right]^{(m)} d z= & -\int_{-\infty}^{\infty}\left[c \bar{b}_{z}^{(r)}(r, z, s)\right]^{(m)} d z \\
& -\frac{Q \lambda(s) r_{0}^{m} r^{m}}{m \pi \epsilon_{0}}\left(\frac{1}{a_{\mathrm{in}}^{2 m}}-\frac{1}{a_{\mathrm{out}}^{2 m}}\right),
\end{aligned}
$$

respectively.

The longitudinal wake potential is given by Eq. (21). By replacing one of $\left[\bar{e}_{z}^{(r)}\right]^{(m)}$ using Eq. (42), we can rewrite this wake potential by the integration of $D^{(m)}$. Then, we obtain 


$$
\begin{aligned}
W_{z}^{(m)}(r, \theta, s)= & -\frac{r^{m} \cos m \theta}{2 Q}\left[\int_{\mathcal{L}_{r}} d z^{\prime} D^{(m)}\left(r, z^{\prime}, s\right)\right. \\
& \left.+\frac{Q \lambda(s) r_{0}^{m}}{a_{\mathrm{out}}^{2 m} m \pi \epsilon_{0}}\left(1-\frac{a_{\mathrm{out}}^{2 m}}{a_{\mathrm{in}}^{2 m}}\right)\right] .
\end{aligned}
$$

The integration path of the first term can be deformed to the contour $\overline{\mathcal{C}}$ by choosing Napoly-Zotter contour $\overline{\mathcal{C}}$ as the contour $\mathcal{C}$ in Eq. (40). The second term of Eq. (43) can be also rewritten as the integration over the contour $\overline{\mathcal{C}}$ by using Eq. (39) and taking the contour $\mathcal{C}$ to be $\overline{\mathcal{C}}$. The wake potential is then expressed as

$$
\begin{aligned}
W_{z}^{(m)}(r, \theta, s)= & -\frac{\cos m \theta}{2 Q} r^{m}\left(\int_{\overline{\mathcal{C}}} D^{(m)}\left(r^{\prime}, z^{\prime}, s\right) d l^{\prime}+\frac{1}{a_{\mathrm{out}}^{2 m}} \int_{\overline{\mathcal{C}}} S^{(m)}\left(r^{\prime}, z^{\prime}, s\right) d l^{\prime}\right) \\
= & -\frac{\cos m \theta}{2 Q} \frac{r^{m}}{a_{\mathrm{out}}^{m}}\left\{\int_{\overline{\mathcal{C}}} d z^{\prime}\left[\bar{e}_{z}^{(r)}\left(\frac{a_{\mathrm{out}}^{m}}{r^{\prime m}}+\frac{r^{\prime m}}{a_{\mathrm{out}}^{m}}\right)-c \bar{b}_{z}^{(r)}\left(\frac{a_{\mathrm{out}}^{m}}{r^{\prime m}}-\frac{r^{\prime m}}{a_{\mathrm{out}}^{m}}\right)\right]^{(m)}\right. \\
& \left.+\int_{\overline{\mathcal{C}}} d r^{\prime}\left[\left(\bar{e}_{r}^{(r)}+c \bar{b}_{\theta}^{(r)}\right)\left(\frac{a_{\mathrm{out}}^{m}}{r^{\prime m}}+\frac{r^{\prime m}}{a_{\mathrm{out}}^{m}}\right)+\left(\bar{e}_{\theta}^{(r)}-c \bar{b}_{r}^{(r)}\right)\left(\frac{a_{\mathrm{out}}^{m}}{r^{\prime m}}-\frac{r^{\prime m}}{a_{\mathrm{out}}^{m}}\right)\right]^{(m)}\right\} .
\end{aligned}
$$

Similarly to Sec. III A, the longitudinal wake potential is expressed in terms of the real fields as

$$
\begin{aligned}
W_{z}^{(m)}(r, \theta, s)= & -\frac{\cos m \theta}{2 Q} \frac{r^{m}}{a_{\mathrm{out}}^{m}}\left\{\int_{\overline{\mathcal{C}}} d z^{\prime}\left[\bar{e}_{z}\left(\frac{a_{\mathrm{out}}^{m}}{r^{\prime m}}+\frac{r^{\prime m}}{a_{\mathrm{out}}^{m}}\right)-c \bar{b}_{z}\left(\frac{a_{\mathrm{out}}^{m}}{r^{\prime m}}-\frac{r^{\prime m}}{a_{\mathrm{out}}^{m}}\right)\right]^{(m)}\right. \\
& \left.+\int_{\overline{\mathcal{C}}} d r^{\prime}\left[\left(\bar{e}_{r}+c \bar{b}_{\theta}\right)\left(\frac{a_{\mathrm{out}}^{m}}{r^{\prime m}}+\frac{r^{\prime m}}{a_{\mathrm{out}}^{m}}\right)+\left(\bar{e}_{\theta}-c \bar{b}_{r}\right)\left(\frac{a_{\mathrm{out}}^{m}}{r^{\prime m}}-\frac{r^{\prime m}}{a_{\mathrm{out}}^{m}}\right)\right]^{(m)}\right\} .
\end{aligned}
$$

The formula (45) looks very similar to formula (24), except that the common beam pipe radius $a$ is replaced by one of the smallest among the two beam pipes radii. Because of that, the integration over $z^{\prime}$ starts from the minus infinity to $z_{2}$ at radial offset $a_{\text {out }}$.

Our task is clearly to put back this integration over infinity to one over a finite length. For this purpose, let us introduce the closed loop $H$ shown in Fig. 3 (the contour $H$ consists of a straight line $\mathcal{D}$ of radius $a_{\text {out }}$ from $z=-\infty$ to $z=z_{1}$ and $\overline{\mathcal{D}}$ of radius $a_{\text {in }}$ from $z=-\infty$ to $z=z_{1}$ and the two radial lines connecting between them at $z=-\infty$ and $z=z_{1}$ ). The loop integrations of $S^{(m)}$ and $D^{(m)}$ over $H$ give us the following identities:

$$
\begin{gathered}
\int_{\overline{\mathcal{D}}} S^{(m)}\left[a_{\mathrm{in}}, z, s\right] d l=\int_{\mathcal{D}} S^{(m)}\left[a_{\mathrm{out}}, z, s\right] d l-\int_{a_{\mathrm{out}}}^{a_{\mathrm{in}}} d r r^{m} \frac{2 Q}{\pi r \epsilon_{0}} \lambda(s)\left(\frac{r r_{0}}{a_{\mathrm{in}}^{2}}\right)^{m}+\left.\int_{a_{\mathrm{out}}}^{a_{\mathrm{in}}} d r r^{m}\left[\bar{e}_{r}^{(r)}+c \bar{b}_{\theta}^{(r)}-\bar{e}_{\theta}^{(r)}+c \bar{b}_{r}^{(r)}\right]^{(m)}\right|_{z=z_{1}}, \\
\int_{\overline{\mathcal{D}}} D^{(m)}\left[a_{\mathrm{in}}, z, s\right] d l=\int_{\mathcal{D}} D^{(m)}\left[a_{\mathrm{out}}, z, s\right] d l+\left.\int_{a_{\mathrm{out}}}^{a_{\mathrm{in}}} d r r^{-m}\left[\bar{e}_{r}^{(r)}+c \bar{b}_{\theta}^{(r)}+\bar{e}_{\theta}^{(r)}-c \bar{b}_{r}^{(r)}\right]^{(m)}\right|_{z=z_{1}}
\end{gathered}
$$

If we choose the contour $\mathcal{D}$ to be the beam chamber axis, we obtain another relation:

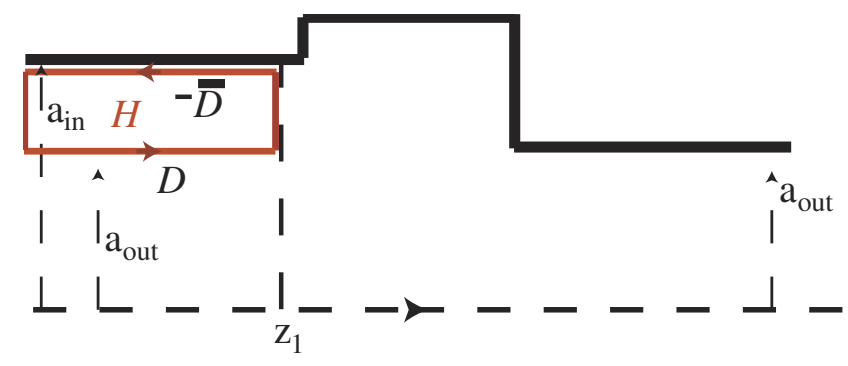

FIG. 3. (Color) The cavity and the integration path. A closed loop $H$ consists of a straight line $\mathcal{D}$ and $\overline{\mathcal{D}}$ and the two radial lines connecting between them at $z=-\infty$ and $z=z_{1}$. The sign of contour $-\overline{\mathcal{D}}$ means that the direction of the integration path is opposite to $\overline{\mathcal{D}}$. The contour $H$ defines the loop path described in the red line. 


$$
\left.\int_{-\infty}^{z_{1}} d z c\left[\bar{b}_{z}^{(r)}\right]^{(m)}\right|_{r=a_{\mathrm{in}}}=-\int_{0}^{a_{\mathrm{in}}} d r r^{m} \frac{2 Q}{\pi r \epsilon_{0}} \frac{\lambda(s)}{a_{\mathrm{in}}^{m}}\left(\frac{r r_{0}}{a_{\mathrm{in}}^{2}}\right)^{m}+\left.\int_{0}^{a_{\mathrm{in}}} d r \frac{r^{m}}{a_{\mathrm{in}}^{m}}\left[\bar{e}_{r}^{(r)}+c \bar{b}_{\theta}^{(r)}-\bar{e}_{\theta}^{(r)}+c \bar{b}_{r}^{(r)}\right]^{(m)}\right|_{z=z_{1}} .
$$

By using Eqs. (46)-(48), we find that the integration of $\bar{e}_{z}$ over $z$ between $-\infty$ and $z_{1}$ can be expressed as

$$
\begin{aligned}
\left.\frac{2}{a_{\text {out }}^{m}} \int_{-\infty}^{z_{1}} d z\left[\bar{e}_{z}\right]^{(m)}\right|_{r=a_{\text {out }}}= & \left.\left(\frac{1}{a_{\text {out }}^{2 m}}-\frac{1}{a_{\text {in }}^{2 m}}\right) \int_{0}^{a_{\text {in }}} d r r^{m}\left[\bar{e}_{r}+c \bar{b}_{\theta}-\bar{e}_{\theta}+c \bar{b}_{r}\right]^{(m)}\right|_{z=z_{1}} \\
& -\left.\int_{a_{\text {out }}}^{a_{\text {in }}} d r \frac{r^{m}}{a_{\text {out }}^{2 m}}\left[\bar{e}_{r}+c \bar{b}_{\theta}-\bar{e}_{\theta}+c \bar{b}_{r}\right]^{(m)}\right|_{z=z_{1}}-\left.\int_{a_{\text {out }}}^{a_{\text {ou }}} d r \frac{1}{r^{m}}\left[\bar{e}_{r}+c \bar{b}_{\theta}+\bar{e}_{\theta}-c \bar{b}_{r}\right]^{(m)}\right|_{z=z_{1}} \\
& +\frac{2 Q}{m \pi \epsilon_{0}} \lambda(s) r_{0}^{m}\left(\frac{1}{a_{\text {out }}^{2 m}}-\frac{1}{a_{\text {in }}^{2 m}}\right) .
\end{aligned}
$$

In Eq. (45), the integration of $\bar{b}_{z}$ fields over the contour $\overline{\mathcal{C}}$ vanishes by taking $r^{\prime}=a_{\text {out }}$, and the integration of $\bar{e}_{z}$ fields over the contour $\overline{\mathcal{C}}$ can be replaced by Eq. (49). Then we obtain for $a_{\text {in }} \geq a_{\text {out }}$ as

$$
\begin{aligned}
W_{z}^{(m)}(r, \theta, s)= & -\frac{\cos m \theta}{2 Q} r^{m}\left\{\frac{Q}{\pi \epsilon_{0}} \lambda(s) \frac{r_{0}^{m}}{m}\left(\frac{1}{a_{\mathrm{out}}^{2 m}}-\frac{1}{a_{\mathrm{in}}^{2 m}}\right)+\frac{1}{a_{\mathrm{out}}^{m}} \int_{z_{1}}^{z_{2}} d z\left[\left(\frac{a_{0}^{m}}{a_{\mathrm{out}}^{m}}+\frac{a_{\mathrm{out}}^{m}}{a_{0}^{m}}\right)\left[\bar{e}_{z}\right]^{(m)}+\left(\frac{a_{0}^{m}}{a_{\mathrm{out}}^{m}}-\frac{a_{\mathrm{out}}^{m}}{a_{0}^{m}}\right)\left[c \bar{b}_{z}\right]^{(m)}\right]\right. \\
& +\left.\left(\frac{1}{a_{\mathrm{out}}^{2 m}}-\frac{1}{a_{\mathrm{in}}^{2 m}}\right) \int_{0}^{a_{0}} d r^{\prime} r^{\prime m}\left[\bar{e}_{r}+c \bar{b}_{\theta}-\bar{e}_{\theta}+c \bar{b}_{r}\right]^{(m)}\right|_{z=z_{1}} \\
& +\left.\int_{a_{\mathrm{in}}}^{a_{0}} d r^{\prime}\left(\frac{\left[\bar{e}_{r}+c \bar{b}_{\theta}+\bar{e}_{\theta}-c \bar{b}_{r}\right]^{(m)}}{r^{\prime m}}+\frac{r^{\prime m}\left[\bar{e}_{r}+c \bar{b}_{\theta}-\bar{e}_{\theta}+c \bar{b}_{r}\right]^{(m)}}{a_{\mathrm{in}}^{2 m}}\right)\right|_{z=z_{1}} \\
& \left.+\left.\int_{a_{0}}^{a_{\mathrm{out}}} d r^{\prime}\left(\frac{\left[\bar{e}_{r}+c \bar{b}_{\theta}+\bar{e}_{\theta}-c \bar{b}_{r}\right]^{(m)}}{r^{\prime m}}+\frac{r^{\prime m}\left[\bar{e}_{r}+c \bar{b}_{\theta}-\bar{e}_{\theta}+c \bar{b}_{r}\right]^{(m)}}{a_{\mathrm{out}}^{2 m}}\right)\right|_{z=z_{2}}\right\} .
\end{aligned}
$$

In this expression, the contribution from the source fields is explicitly written in the first term. We should notice that the $z$-integration over infinity in Eq. (45) is replaced by that over the finite length in Eq. (50).

The similar argument can be made for the case that $a_{\text {out }}$ is larger than $a_{\text {in }}$. The detail is described in Appendix B. The general expression of the longitudinal wake potential (which can be applied to not only the case $a_{\text {out }} \leq a_{\text {in }}$ but also to the case $\left.a_{\mathrm{out}} \geq a_{\mathrm{in}}\right)$ is given by

$$
\begin{aligned}
W_{z}^{(m)}(r, \theta, s)= & -\frac{\cos m \theta}{2 Q} r^{m}\left\{\frac{Q}{\pi \epsilon_{0}} \lambda(s) \frac{r_{0}^{m}}{m}\left(\frac{1}{a_{\mathrm{out}}^{2 m}}-\frac{1}{a_{\mathrm{in}}^{2 m}}\right)+\frac{1}{a_{\mathrm{min}}^{m}} \int_{z_{1}}^{z_{2}} d z\left[\left(\frac{a_{0}^{m}}{a_{\text {min }}^{m}}+\frac{a_{\mathrm{min}}^{m}}{a_{0}^{m}}\right)\left[\bar{e}_{z}\right]^{(m)}+\left(\frac{a_{0}^{m}}{a_{\min }^{m}}-\frac{a_{\mathrm{min}}^{m}}{a_{0}^{m}}\right)\left[c \bar{b}_{z}\right]^{(m)}\right]\right. \\
& +\left.\left(\frac{1}{a_{\mathrm{out}}^{2 m}}-\frac{1}{a_{\mathrm{in}}^{2 m}}\right) \int_{0}^{a_{0}} d r^{\prime} r^{\prime m}\left[\bar{e}_{r}+c \bar{b}_{\theta}-\bar{e}_{\theta}+c \bar{b}_{r}\right]^{(m)}\right|_{z=z_{j}} \\
& +\left.\int_{a_{\mathrm{in}}}^{a_{0}} d r^{\prime}\left(\frac{\left[\bar{e}_{r}+c \bar{b}_{\theta}+\bar{e}_{\theta}-c \bar{b}_{r}\right]^{(m)}}{r^{\prime m}}+\frac{r^{\prime m}\left[\bar{e}_{r}+c \bar{b}_{\theta}-\bar{e}_{\theta}+c \bar{b}_{r}\right]^{(m)}}{a_{\mathrm{in}}^{2 m}}\right)\right|_{z=z_{1}} \\
& \left.+\left.\int_{a_{0}}^{a_{\mathrm{out}}} d r^{\prime}\left(\frac{\left[\bar{e}_{r}+c \bar{b}_{\theta}+\bar{e}_{\theta}-c \bar{b}_{r}\right]^{(m)}}{r^{\prime m}}+\frac{r^{\prime m}\left[\bar{e}_{r}+c \bar{b}_{\theta}-\bar{e}_{\theta}+c \bar{b}_{r}\right]^{(m)}}{a_{\mathrm{out}}^{2 m}}\right)\right|_{z=z_{2}}\right\}
\end{aligned}
$$

where the notation $a_{\text {min }}$ is $\min \left(a_{\text {in }}, a_{\text {out }}\right), z_{j}$ is $z_{1}$ in the case of $a_{\mathrm{out}} \leq a_{\mathrm{in}}$, and that is $z_{2}$ in the case of $a_{\mathrm{out}} \geq a_{\mathrm{in}}$. When the radius $a_{\text {in }}$ is equal to the radius $a_{\text {out }}$, Eq. (51) reproduces the previous result of Napoly integral, given by Eq. (24).

\section{APPLICATIONS : THE IMPLEMENTATION TO ABCI CODE}

The new Eq. (51), which is the extension of Napoly integral, has been implemented into ABCI code [1]. Even before the implementation of this new formula, $\mathrm{ABCI}$ ac- tually could compute the transverse wake potential of the dipole fields using the conventional integration method on a straight line for a structure with unequal beam pipes radii and correct the difference of the potential energies in the two beam pipes at both ends. The problem of this method is naturally that very long beam pipes, in particular, on the outgoing side, may be necessary to simulate the interaction between a beam and the wakefields accurately. Equation (51) permits the integration path can be confined to a finite length by having the integration contour beginning and ending on the beam tubes. 

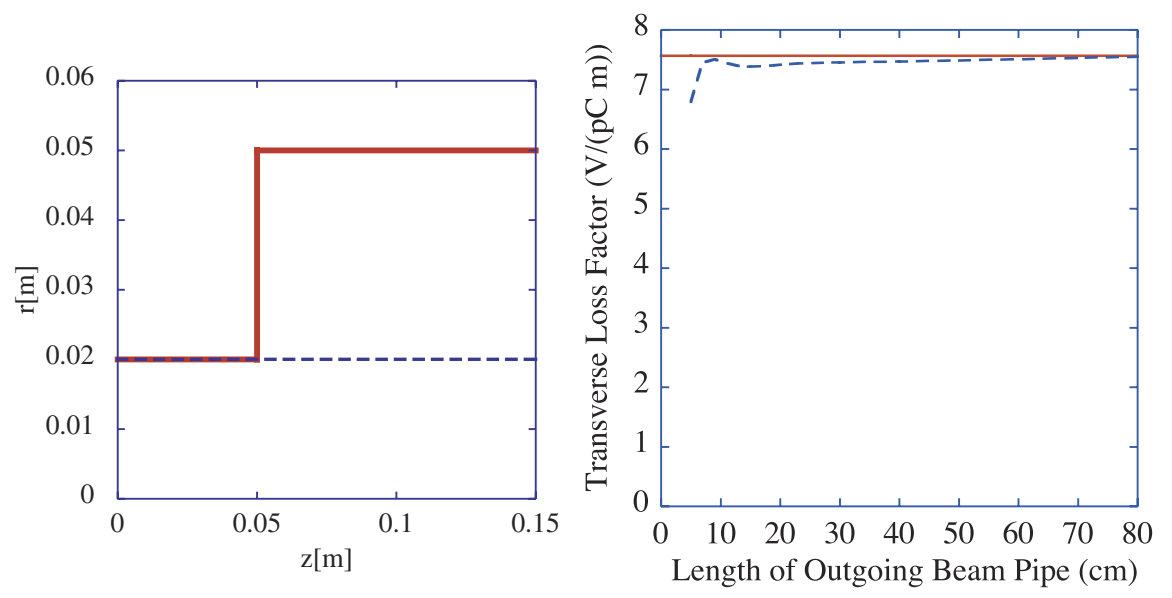

FIG. 4. (Color) Comparison between the present method and the conventional integration methods. The dashed straight line in the left figure shows the integration path for the conventional integration method. The horizontal axis of the right figure shows the length of the outgoing beam pipe. The red line represents the kick factor obtained by the present method, and the blue dashed line does that by the conventional method. About 20 times longer beam pipe than the aperture of the pipe is necessary to calculate the transverse wake potential accurately.

To see how quickly the calculation result converges as a function of the outgoing beam pipe length in the conventional method, we compare the transverse loss (kick) factor:

$$
\kappa=\int_{0}^{\infty} d f 2 \Im\left[Z_{\perp}(f)\right] e^{-4 \pi^{2} f^{2} \sigma_{t}^{2}}
$$

(where $\sigma_{t}$ is the bunch length in time) of the our new integration method with that of the conventional integration method for a step-out structure shown in the left part of Fig. 4. The dashed straight line in this figure shows the integration path for the conventional integration method. The bunch length $c \cdot \sigma_{t}$ is $2 \mathrm{~cm}$. The right panel of Fig. 4 shows the comparison result. The horizontal axis shows the length of the outgoing beam pipe. We can see that about 20 times longer beam pipe than the aperture of the pipe is necessary to calculate the transverse wake potential accurately.

\section{SUMMARY}

By using the closed one-forms defined on the $(r, z)$-plane, we generalize the Napoly integral for any azimuthal mode, $m$, and for any structure. In their original Napoly integral, it is not necessary to deal with the contribution of the radiated fields at both ends of the beam tube, because the structure has equal radii at both ends. Further, they did not have to care about the contribution from the source fields, because that becomes zero by applying the Napoly-Zotter contour in their integration. We have completed the Napoly integral by considering those effects for the structure that has unequal radii of the beam tube at both ends.

Equation (51) permits calculations of wake potentials in structures extending to the inside of the beam tube radius or having unequal tube radii at the two sides not only for longitudinal but also for transverse cases, while the integration path can be confined to a finite length by having the integration contour beginning and ending on the beam tubes.

The extended method has been implemented to the $\mathrm{ABCI}$ code. ABCI automatically finds the best-deformed contour for the integral. Users can now input any type of structure, and $\mathrm{ABCI}$ always provides accurate results.

\section{ACKNOWLEDGMENTS}

The authors thank T. Toyama for discussions. The authors also thank the members of the J-PARC project at JAEA/KEK.

\section{APPENDIX A: THE MONOPOLE $(m=0)$ LONGITUDINAL WAKE POTENTIALS}

In the monopole mode, two vectors defined by Eqs. (9) and (10) can be combined as

$$
\tilde{S}^{(m=0)}=\left(\left[\bar{e}_{r}^{(r)}+c \bar{b}_{\theta}^{(r)}\right]^{m=0},\left[\bar{e}_{z}^{(r)}\right]^{m=0}\right) .
$$

Let us consider an axisymmetric cavity as shown in Fig. 5. The radius of the beam tube on the downstream side is $a_{\text {out }}$ and that on the upstream side is $a_{\text {in }}$. We choose a closed loop $G$ (see Fig. 5) in such a way that it consists of a straight line $\mathcal{L}_{r}$ and an arbitrary contour $\mathcal{C}$ and the two radial lines connecting between them at $z= \pm \infty$. The contour $\mathcal{C}$ has radial offsets $r_{\text {in }}$ and $r_{\text {out }}$ at $z= \pm \infty$, respectively.

Since the asymptotic forms of the radiated fields in the $m=0$ mode vanish for $z \rightarrow \pm \infty$, the integrations along the radial lines at $z= \pm \infty$ have no contribution. Then, the longitudinal wake potential can be written as 


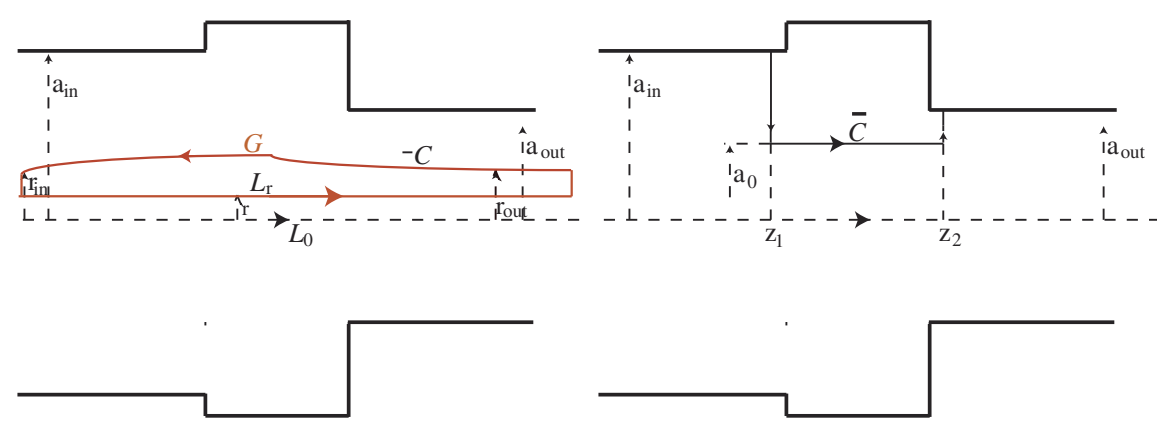

FIG. 5. (Color) The cavity and the integration path. The beam tube of the downstream side is denoted as $a_{\text {out }}$ and the upstream side $a_{\text {in }}$. A closed loop $G$ in the left figure consists of a straight line $\mathcal{L}_{r}$ and arbitrary contour $\mathcal{C}$ and the two radial lines connecting between them at $z= \pm \infty$. The contour $\mathcal{C}$ has radial offsets $r_{\text {in }}$ and $r_{\text {out }}$ at $z= \pm \infty$, respectively. The sign of contour $-\mathcal{C}$ means that the direction of the integration path is opposite to $\mathcal{C}$. The contour $\overline{\mathcal{C}}$ in the right figure which is composed of three longitudinal line segments at different radii connected by two radial segments is called "Napoly-Zotter contour" [3].

$$
\begin{aligned}
W_{z}^{(m=0)} & =-\frac{1}{Q} \int_{-\infty}^{\infty} d z\left[\bar{e}_{z}\right]^{(m=0)} \\
& =-\frac{1}{Q} \int_{\mathcal{C}}\left(d z\left[\bar{e}_{z}^{(r)}\right]^{(m=0)}+d r\left[\bar{e}_{r}^{(r)}+c \bar{b}_{\theta}^{(r)}\right]^{m=0}\right)
\end{aligned}
$$

By replacing the radiated fields of Eq. (A2) by the real fields, which can be calculated from

$$
\begin{gathered}
\phi^{(s)}(r, \theta, z, t)=\frac{Q}{2 \pi \epsilon_{0}} \lambda(c t-z) \log \left(\frac{a_{\mathrm{out}}}{r_{>}}\right), \\
A_{z}^{(s)}(r, \theta, z, t)=\phi^{(s)}(r, \theta, z, t) / c,
\end{gathered}
$$

the general expression of the monopole wake potential can be obtained as

$$
\begin{aligned}
W_{z}^{(m=0)}= & -\frac{1}{Q} \int_{\mathcal{C}}\left(d z\left[\bar{e}_{z}\right]^{(m=0)}+d r\left[\bar{e}_{r}+c \bar{b}_{\theta}\right]^{m=0}\right) \\
& +\frac{\lambda(s)}{\pi \epsilon_{0}} \ln \frac{r_{\text {out }}}{r_{\text {in }}} .
\end{aligned}
$$

It is convenient to choose the contour $\overline{\mathcal{C}}$ such that it starts and ends on the beam pipe on both ends. In this case, the integrations along the tubes vanish $\left(E_{z}=0\right)$ and the integration along the contour $\mathcal{C}$ is confined to a finite length. In particular, the contour, which is composed of three longitudinal line segments at different radii connected by two radial segments, is called "Napoly-Zotter contour" and is denoted by the contour $\overline{\mathcal{C}}$ in the right part of Fig. 5. In this particular case, the formula for longitudinal wake potentials becomes

$$
\begin{aligned}
W_{z}^{(m=0)}= & -\frac{1}{Q} \int_{\overline{\mathcal{C}}}\left(d z\left[\bar{e}_{z}\right]^{(m=0)}+d r\left[\bar{e}_{r}+c \bar{b}_{\theta}\right]^{m=0}\right) \\
& +\frac{\lambda(s)}{\pi \epsilon_{0}} \ln \frac{a_{\text {out }}}{a_{\text {in }}} .
\end{aligned}
$$

The second term represents the contribution from the source field.

\section{APPENDIX B: FORMULA OF WAKE POTENTIAL FOR $a_{\text {in }} \leq a_{\text {out }}$}

A similar argument can be made for the case that the tube radius on the downstream side $a_{\text {out }}$ is larger than that on the upstream side $a_{\text {in }}$. The contour $\overline{\mathcal{C}}$ needs be slightly modified from the left part of Fig. 2 to one shown in Fig. 6 (the radial offset at both chambers is now $a_{\text {in }}$ instead of $\left.a_{\text {out }}\right)$. The asymptotic forms of the radiated fields [Eqs. (35)-(38)] are unchanged by defining the source field as

$$
\begin{aligned}
\phi^{(s)}(r, \theta, z, t)= & \frac{Q}{2 \pi \epsilon_{0}} \lambda(c t-z)\left[\log \left(\frac{a_{\text {in }}}{r_{>}}\right)\right. \\
& \left.+\sum_{m=1}^{\infty} \frac{\cos m \theta}{m}\left(\frac{r_{<}}{r_{>}}\right)^{m}\right],
\end{aligned}
$$

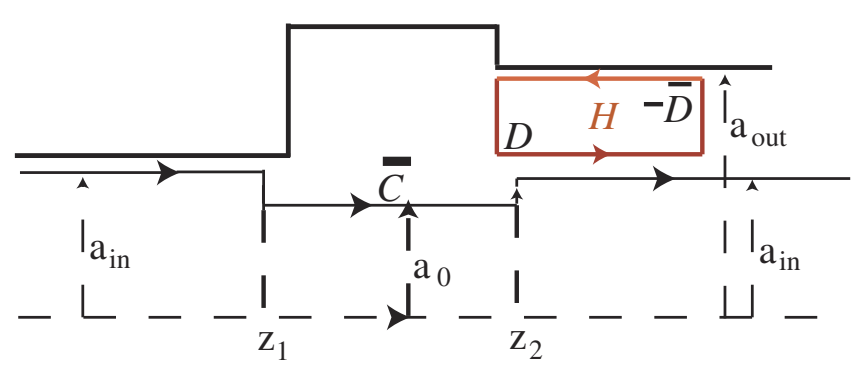

FIG. 6. (Color) The cavity and the integration path. Here the tube radius of the downstream side $a_{\text {out }}$ is larger than that of the upstream side $a_{\text {in }}$. The longitudinal coordinate $z_{1}$ and $z_{2}$ and the radial coordinate $a_{0}$ specify the contour $\overline{\mathcal{C}}$. The contour $H$ defines the loop path described in the red line. 


$$
A_{z}^{(s)}(r, \theta, z, t)=\phi^{(s)}(r, \theta, z, t) / c,
$$

where $r_{>}=\sup \left(r, r_{0}\right)$ and $r_{<}=\inf \left(r, r_{0}\right)$.

It is straightforward to understand that the expression of wake potential along the contour $\overline{\mathcal{C}}$ is the one obtained by replacing $a_{\text {out }}$ in Eqs. (44) and (45) by $a_{\text {in }}$. Namely,

$$
\begin{aligned}
W_{z}^{(m)}(r, \theta, s)= & -\frac{\cos m \theta}{2 Q} r^{m}\left(\int_{\overline{\mathcal{C}}} D^{(m)}\left(r^{\prime}, z^{\prime}, s\right) d l^{\prime}+\frac{1}{a_{\mathrm{in}}^{2 m}} \int_{\overline{\mathcal{C}}} S^{(m)}\left(r^{\prime}, z^{\prime}, s\right) d l^{\prime}\right) \\
= & -\frac{\cos m \theta}{2 Q} \frac{r^{m}}{a_{\mathrm{in}}^{m}}\left\{\int_{\overline{\mathcal{C}}} d z^{\prime}\left[\bar{e}_{z}\left(\frac{a_{\mathrm{in}}^{m}}{r^{\prime m}}+\frac{r^{\prime m}}{a_{\mathrm{in}}^{m}}\right)-c \bar{b}_{z}\left(\frac{a_{\mathrm{in}}^{m}}{r^{\prime m}}-\frac{r^{\prime m}}{a_{\mathrm{in}}^{m}}\right)\right]^{(m)}\right. \\
& \left.+\int_{\overline{\mathcal{C}}} d r^{\prime}\left[\left(\bar{e}_{r}+c \bar{b}_{\theta}\right)\left(\frac{a_{\mathrm{in}}^{m}}{r^{\prime m}}+\frac{r^{\prime m}}{a_{\mathrm{in}}^{m}}\right)+\left(\bar{e}_{\theta}-c \bar{b}_{r}\right)\left(\frac{a_{\mathrm{in}}^{m}}{r^{\prime m}}-\frac{r^{\prime m}}{a_{\mathrm{in}}^{m}}\right)\right]^{(m)}\right\} .
\end{aligned}
$$

Again, we have the integration over infinity, but this time from $z=z_{2}$ to infinity, instead of from minus infinity to $z=z_{1}$.

This integration can be replaced by one over a finite length by introducing the closed loop $H$ shown in Fig. 6. (The contour $H$ consists of a straight line $\mathcal{D}$ of radius $a_{\text {in }}$ from $z=z_{2}$ to $z=\infty$ and $\overline{\mathcal{D}}$ of radius $a_{\text {out }}$ from $z=z_{2}$ to $z=\infty$ and the two radial lines connecting between them at $z=z_{2}$ and $z=\infty$.) The loop integrations of $S^{(m)}$ and $D^{(m)}$ over $H$ give us the following identities:

$$
\begin{array}{r}
\int_{\overline{\mathcal{D}}} S^{(m)}\left[a_{\mathrm{out}}, z, s\right] d l=\int_{\mathcal{D}} S^{(m)}\left[a_{\mathrm{in}}, z, s\right] d l-\int_{a_{\mathrm{out}}}^{a_{\mathrm{in}}} d r r^{m} \frac{2 Q}{\pi r \epsilon_{0}} \lambda(s)\left(\frac{r r_{0}}{a_{\text {out }}^{2}}\right)^{m}+\left.\int_{a_{\mathrm{out}}}^{a_{\mathrm{in}}} d r r^{m}\left[\bar{e}_{r}^{(r)}+c \bar{b}_{\theta}^{(r)}-\bar{e}_{\theta}^{(r)}+c \bar{b}_{r}^{(r)}\right]^{(m)}\right|_{z=z_{2}}, \\
\int_{\overline{\mathcal{D}}} D^{(m)}\left[a_{\mathrm{out}}, z, s\right] d l=\int_{\mathcal{D} 4)} D^{(m)}\left[a_{\mathrm{in}}, z, s\right] d l+\left.\int_{a_{\mathrm{out}}}^{a_{\mathrm{in}}} d r r^{-m}\left[\bar{e}_{r}^{(r)}+c \bar{b}_{\theta}^{(r)}+\bar{e}_{\theta}^{(r)}-c \bar{b}_{r}^{(r)}\right]^{(m)}\right|_{z=z_{2}} \cdot(\mathrm{B} 5)
\end{array}
$$

By choosing the contour $\mathcal{D}$ to be the beam axis, we obtain another relation:

$$
\left.\int_{z_{2}}^{\infty} d z c\left[\bar{b}_{z}^{(r)}\right]^{(m)}\right|_{r=a_{\text {out }}}=\int_{0}^{a_{\text {out }}} d r r^{m} \frac{2 Q}{\pi r \epsilon_{0}} \frac{\lambda(s)}{a_{\text {out }}^{m}}\left(\frac{r r_{0}}{a_{\text {out }}^{2}}\right)^{m}-\left.\int_{0}^{a_{\text {out }}} d r \frac{r^{m}}{a_{\text {out }}^{m}}\left[\bar{e}_{r}^{(r)}+c \bar{b}_{\theta}^{(r)}-\bar{e}_{\theta}^{(r)}+c \bar{b}_{r}^{(r)}\right]^{(m)}\right|_{z=z_{2}} .
$$

Following the same manipulation as for the $a_{\text {out }} \leq a_{\text {in }}$ case, we can easily identify that the $\bar{e}_{z}$ integration over infinity can be expressed by ones over finite length:

$$
\begin{aligned}
\left.\frac{2}{a_{\mathrm{in}}^{m}} \int_{z_{2}}^{\infty} d z\left[\bar{e}_{z}\right]^{(m)}\right|_{r=a_{\text {in }}}= & \left.\left(\frac{1}{a_{\mathrm{out}}^{2 m}}-\frac{1}{a_{\mathrm{in}}^{2 m}}\right) \int_{0}^{a_{\mathrm{in}}} d r r^{m}\left[\bar{e}_{r}+c \bar{b}_{\theta}-\bar{e}_{\theta}+c \bar{b}_{r}\right]^{(m)}\right|_{z=z_{2}} \\
& -\left.\int_{a_{\mathrm{out}}}^{a_{\mathrm{in}}} d r \frac{r^{m}}{a_{\mathrm{in}}^{2 m}}\left[\bar{e}_{r}+c \bar{b}_{\theta}-\bar{e}_{\theta}+c \bar{b}_{r}\right]^{(m)}\right|_{z=z_{2}}-\left.\int_{a_{\mathrm{out}}}^{a_{\mathrm{in}}} d r \frac{1}{r^{m}}\left[\bar{e}_{r}+c \bar{b}_{\theta}+\bar{e}_{\theta}-c \bar{b}_{r}\right]^{(m)}\right|_{z=z_{2}} \\
& +\frac{2 Q}{m \pi \epsilon_{0}} \lambda(s) r_{0}^{m}\left(\frac{1}{a_{\mathrm{out}}^{2 m}}-\frac{1}{a_{\mathrm{in}}^{2 m}}\right)
\end{aligned}
$$

By inserting Eq. (B7) into Eq. (B3) and taking $r^{\prime}=a_{\text {in }}$ in the first integration, we can reach an expression of wake potential for $a_{\text {in }} \leq a_{\text {out }}$ as

$$
\begin{aligned}
W_{z}^{(m)}(r, \theta, s)= & -\frac{\cos m \theta}{2 Q} r^{m}\left\{\frac{Q}{\pi \epsilon_{0}} \lambda(s) \frac{r_{0}^{m}}{m}\left(\frac{1}{a_{\mathrm{out}}^{2 m}}-\frac{1}{a_{\mathrm{in}}^{2 m}}\right)+\frac{1}{a_{\mathrm{in}}^{m}} \int_{z_{1}}^{z_{2}} d z\left[\left(\frac{a_{0}^{m}}{a_{\mathrm{in}}^{m}}+\frac{a_{\mathrm{in}}^{m}}{a_{0}^{m}}\right)\left[\bar{e}_{z}\right]^{(m)}+\left(\frac{a_{0}^{m}}{a_{\mathrm{in}}^{m}}-\frac{a_{\mathrm{in}}^{m}}{a_{0}^{m}}\right)\left[c \bar{b}_{z}\right]^{(m)}\right]\right. \\
& \left.+\left.\left(\frac{1}{a_{\mathrm{out}}^{2 m}}-\frac{1}{a_{\mathrm{in}}^{2 m}}\right) \int_{0}^{a_{0}} d r^{\prime} r^{\prime m}\left[\bar{e}_{r}+c \bar{b}_{\theta}-\bar{e}_{\theta}+c \bar{b}_{r}\right]^{(m)}\right|_{z=z_{2}}\right)\left.\right|_{z=z_{1}} \\
& \left.+\left.\int_{a_{\mathrm{in}}}^{a_{0}} d r^{\prime}\left(\frac{\left[\bar{e}_{r}+c \bar{b}_{\theta}+\bar{e}_{\theta}-c \bar{b}_{r}\right]^{(m)}}{r^{\prime m}}+\frac{r^{\prime m}\left[\bar{e}_{r}+c \bar{b}_{\theta}-\bar{e}_{\theta}+c \bar{b}_{r}\right]^{(m)}}{a_{\mathrm{in}}^{2 m}}\right)\right|_{z=z_{2}}\right\} . \\
& +\int_{a_{0}}^{a_{\text {out }}} d r^{\prime}\left(\frac{\left[\bar{e}_{r}+c \bar{b}_{\theta}+\bar{e}_{\theta}-c \bar{b}_{r}\right]^{(m)}}{r^{\prime m}}+\frac{r^{\prime m}\left[\bar{e}_{r}+c \bar{b}_{\theta}-\bar{e}_{\theta}+c \bar{b}_{r}\right]^{(m)}}{a_{\text {out }}^{2 m}}\right) \mid
\end{aligned}
$$


By combining the two formulas [Eqs. (50) and (B8)], we can get the united expression, Eq. (51), good for both $a_{\text {in }} \leq$ $a_{\text {out }}$ and $a_{\text {in }} \geq a_{\text {out }}$ cases.

[1] Y.H. Chin, KEK Report No. 2005-06, 2005, http://wwwlib.kek.jp/cgi-bin/kiss_prepri.v8?KN=200524006\&OF=8, http://abci.kek.jp/abci.htm.
[2] O. Napoly, Part. Accel. 36, 15 (1991).

[3] O. Napoly, Y.H. Chin, and B. Zotter, Nucl. Instrum. Methods Phys. Res., Sect. A 334, 255 (1993).

[4] H. Henke and W. Bruns, in Proceedings of EPAC 2006, Edinburgh, Scotland, p. 2170 (WEPCH110), http:// www.jacow.org/.

[5] W. Panofsky and W. Wenzel, Rev. Sci. Instrum. 27, 967 (1956). 